\title{
Sédentarisation Des Mbororo, Saturation Foncière Dans Le Nord-Ouest Et Difficile Migration vers Bangangté dans l'Ouest-Cameroun
}

\author{
Abdoulay Mfewou, PhD \\ Fon Dorothy Engwali, PhD \\ Youssouf Monkouop, MA \\ University of Dschang, Cameroon
}

\section{Résumé}

La sédentarisation, le Cameroun, que ce soit avant ou après l'indépendance, s'est donné comme objectif de sédentariser ces éleveurs nomades pour mieux les contrôler. Cet article montre qu'après leur ancrage réussir à Bamenda dans la région du Nord-Ouest Cameroun, la nouvelle génération des éleveurs Mbororo peine à se stabiliser dans le département du Ndé (Banganté-Ouest-Cameroun) face au contrôle foncier coutumier des autorités traditionnelles (autochtones). Ici, les notables fonciers contrôlent l'espace et ne souhaitent qu'accorder qu'une hospitalité d'installation nsurveillée et limitée aux éleveurs par rapport à la région du Nord-Ouest Cameroun, où, les Mbororo avaient bénéficié d'hospitalité et d'un accord verbal d'installation et même sont devenus aujourd'hui des propriétés foncières dans cette partie du pays. A Banganté, l'implication politique de l'Etat est presque faible dans l'encadrement et la délimitation d'espaces réservés à l'élevage. Des couloirs de circulation des animaux et d'autres espaces attribués à l'agriculture entrent faiblement dans les politiques agricoles. Il manque jusqu'ici une véritable politique d'aménagement de l'espace et de prévision pour se doter d'une politique d'accompagnement des déplacements des éleveurs vers les savanes vierges.

Mots-clés :Bangangté, foncier, Migration, Ouest-Cameroun, Sédentarisation 


\title{
Mbororo Settlement, Land Saturation in the North-West, and Difficult Migration to Bangangté in West-Cameroon
}

\author{
Abdoulay Mfewou, PhD \\ Fon Dorothy Engwali, PhD \\ Youssouf Monkouop, MA \\ University of Dschang, Cameroon
}

\begin{abstract}
Settlement policies in Cameroon, whether before or after independence, has established goals for the settlement of nomadic herders to better control them. This paper shows that after their successful settlement in Bamenda in the North-West region of Cameroon, the new generation of Mbororo farmers struggled to move and stabilize in the Ndé division (Banganté-West-Cameroon). They were, however, faced with customary land control by traditional authorities. Here, the land owners controlled the land and only donated it to locals. This limited the nomads from acquiring such land in this region as compared to traditional authorities in the North-West that allowed Mbororo herdsmen to benefit from the hospitality, verbal settlement agreement, and even owning property in this part of the country. In Banganté, the political involvement of the state in the supervision and delimitation of land reserved for breeding is dysfunctional. Animal circulation corridors and other areas allocated for agriculture are poorly involved in agricultural policies. Until now, there has been a lack of planning and forecasting policy put in place to develop and support the movement of pastoralists into virgin savannahs.
\end{abstract}

Keywords: Bangangté, land tax, Migration, Ouest-Cameroun

\section{Introduction}

Anciens nomades devenus sédentaires (Dongmo et al., 2012 ; Pelican, 2011; Boutrais, 1984), les éleveurs Mbororo ont occupé de grandes superficies dans la region du Nord-Ouest du Cameroun grâce à l'hospitalité des chefs coutumiers. À partir des années 1920, ils se sont ancrés progressivement dans les Grassfields, avec une faible densité $\left(2-5 \mathrm{hab} / \mathrm{km}^{2}\right)$. À cette époque, la pression foncière était très faible dans ces savanes. 
Dans le Nord-Ouest du Cameroun (17 $300 \mathrm{~km}^{2}$ ), les Mbororo Djafoun puis les Mbororo Akou, en provenance de l'Adamaoua via le «pays » Bamoun, s'installèrent aux environs de Bamenda, une autre partie restante à Didango (Foumban) (Boutrais, 1984). Dans ses travaux, Boutrais (1992; 1984) montre que les Mbororo Djafoun furent les premiers à entrer aux Grassfields. Ils s'installèrent dans des savanes qui relèvent des chefs traditionnels et des héritiers fonciers et, politiquement, de l'Etat à travers la législation foncière du domaine national (Mfewou et al., 2014; Ngengong, 2007).

Ainsi, la sédentarisation des éleveurs est posée au préalable à la modernisation de l'élevage (Exchange, 2010 ; Sado, 2010), au point que celleci s'y confond et, trop souvent, s'y arrêté. Plus encore, celle-ci ne représente peut-être pas le meilleur moyen d'améliorer leurs techniques d'élevage. Enfin, la sédentarisation était jugée par l'administration, compatible avec le progrès technique d'élevage et le développement socio-économique des acteurs (Pemunta et al., 2013 ; Tallet, 2003).

Les entraves rencontrées par les éleveurs Mbororo s'expliquent aussi par leur précarité et de leur rejet de la part des natives. S'y ajoute actuellement l'impact de l'accroissement démographique à Bamenda $\left(99,9 \mathrm{hab} / \mathrm{km}^{2}\right.$; BUCREP, 2011) qui accélère le sentiment de fin de terres disponibles pour la nouvelle génération (autochtones et Mbororo) (Dongmo, 2012 ; Courade, 2007). De plus, les Mbororo ne disposaient pas une organisation sociétale structurée avant la création en 1992 de leur association pour le développement social et culturel des Mbororo (MBOSCUDA) et qui est chargée de défendre leur intêret, leur intégration et leurs droits citoyens. Hier, l'installation des Mbororo était basée sur un accord verbal avec des chefs coutumiers du NordOuest. Les Mbororo ont ainsi pu exploiter des savanes sans limitation d'étendue ni de durée. Cette large hospitalité a contribué à l'arrivée massive des Mbororo dans les années 1950 et 60 aux Grassfields.

Dans le Nord-Ouest Cameroun, l'on enregistre de profondes mutations chez les Mbororo, leur «éveil» (éducation), l'étalement de la ville de Bamenda, l'essor démographique de la nouvelle génération montante, l'augmentation des effectifs du bétail (45000 têtes/zones d'élevages). Les progrès importants des productions agricoles et l'expansion des surfaces cultivées ont réduit les espaces pastoraux, provoquant une saturation foncière et une surcharge en bétail. S'ajoutent à la pression foncière, la diffusion et la dispersion des champs de brousse qui ont accru les dégâts causés par les animaux. Les conflits fonciers récurrents parfois avec mort d'homme dans les deux camps, les conflits fonciers entre agriculteurs et éleveurs se multiplient. Cette forte consommation d'espace soit pour l'élevage extensif, soit pour le bâti, ou pour l'agriculture a entraîné de multiples conflits fonciers entre différents acteurs qui ont remis en cause les mécanismes des anciennes 
modalités d'accès à la terre par donation et hospitalité des chefs coutumiers du Nord-Ouest.

Bien que la référence aux règles coutumières d'accès à la terre des ancêtres reste toujours entre les mains des descendants fonciers, «maîtres de la terre», on note à Bamenda la perte de contrôle après l'installation des éleveurs par les fons (chefs coutumiers), on note l'enregistrement de plusieurs villages d'éleveurs et la reconnaissance des chefferies Mbororo (lamido) par l'administration publique (Didango). Ceci remet aussi en cause des accords antérieurs entre autochtones et éleveurs (accords verbaux). La pratique traditionnelle des dons de terres coutumiers aux Mbororo est presque finie à Bamenda, où certains Mbororo disposent de titres de propriété foncière.

En effet, face à la saturation foncière, quelles sont les stratégies endogènes développées par les Mbororo de Bamenda et la nouvelle génération d'éleveurs qui migrent vers les savanes non exploitées de la région de l'OuestCameroun? Quelles sont les implications du politique dans cette nouvelle migration?

On enregistre des terres propices à l'élevage (Sud-Foumban, Nord de Bangangté et en limites avec la forêt dense vers Bafia région du Centre) accueillent de jeunes éleveurs. Mais une compétition foncière pour les cultures maraîchères y existe déjà entre les autochtones (Mfewou et al., 2014). La rente foncière y étant jugée très grande par les chefs coutumiers, les dons de terres sont remplacés par des locations à durée déterminée.

\section{Méthodologie de recherché}

Situé à $1350 \mathrm{~m}$ d'altitude, d'une moyenne annuelle de précipitation comprise entre 1400 et $2500 \mathrm{~mm}$, inégalement répartie et avec une population de 300000 habitants, Bangangté est le chef-lieu du département du Ndé dans la région de l'Ouest Cameroun, cette zone enregistre des espaces propice à l'agriculture et à l'élevage.

L'étude dans cette zone s'intéresse à la nouvelle génération des éleveurs Mbororo venus de Bamenda, qui migrent et occupent les savanes du département de Ndé à Banganté (Ouest-Cameroun). Notre critère de choix a été porté sur les jeunes migrants Mbororo et sur leur nouvelle unité domestique dans les savanes arborées de Banganté (campement projet, campement Nana, et campement Bangang-Fokam). Un échantillon de 49 familles reparties dans les trois campements choisi à l'aléatoirement a été retenu dans nos enquêtes.

Dans ce contexte de la nouvelle sédentarisation, nous avons aussi fait une autre enquête auprès des autorités foncières locales (autochtones) sur les modalité d'accueil et d'attribution de terre aux éleveurs et enfin, une autre enquête auprès du personnel du ministère en charge du monde rural sur l'implication du politique. 
En définitive, nous avons analysé le rôle des chefs coutumiers du Nord-Ouest au point de départ de Bamenda d'une part, et celui de Bangangté nouveau pâturage comme point d'arrivée d'autre part, l'analyse sur les questions foncières, en s'appuyant sur des archives locales. L'histoire du développement est abordée d'après les critères d'attribution des terres, en tenant compte de l'insécurité foncière et enfin de la médiation foncière apportée par les autorités locales face aux conflits fonciers récurrents enregistrés.

\section{Histoire de développement local des éleveurs Mbororo et gestion coutumière des terres du Nord-Ouest}

D’après les travaux de Boutrais (1984), dans les années 1920, les premiers Mbororo restaient toujours des nomades, « eggo-eggoobe » : ceux qui vont et reviennent, ou wanchoobe : ceux qui se promènent. Leur séjour dans la région du Nord-Ouest ne se traduisit pas aussitôt par une fixation. Il faut noter la présence du premier chef (ardo) qui est resté au même endroit de 1920 jusqu'à sa mort en 1960. Les autres nomades, changeant de pâturage en fonction des saisons presque chaque année. Les va-et-vient entre la région du Nord-Ouest et la région de l'Adamaoua-Mambila étaient incessants. En 1931, l'invasion de la région de l'Adamaoua par les criquets va pousser les mouvements massifs des éleveurs vers le «pays» Bamoun (OuestCameroun).

À la faveur d'installations continuelles, la sédentarisation (joondé) des Mbororo progresse au cours des années 1960 (Boutrais, 1984). A leur arrivée à Bamenda, ces éleveurs ont rencontré de grandes chefferies traditionnelles bien structurées. Conformément au droit coutumier, le chef de terre (souvent le fon), décidant de l'octroi des parcelles foncières aux autochtones, il l'a fait également aux Mbororo. Ceux-ci ont bénéficié d'une hospitalité des chefs coutumiers, ce qui a favorisé leur sédentarisation. Une enquête démographique a ainsi découvert que «la population Mbororo est moins mobile que l'on aurait pu s'y attendre » (cité par Boutrais, 1984). Les éleveurs vont occuper des espaces dans la région du Nord-Ouest sans limitation d'étendue ni limitation de durée sur un accord verbal. Il faut noter qu'en échange, les Mbororo s'engageaient à respecter des interdits locaux (ne pas verser du sang en cas de bagarre, faire des dons d'animaux à la chefferie pour les cérémonies de fin d'année...). C'est sur cette très large hospitalité que les éleveurs Mbororo vont s'installer à Bamenda, acquérir de grandes superficies et devenir des autochtones dans cette partie du pays.

Avec le bon accueil par les autochtones, le mode de vie des familles Mbororo va changer rapidement à la faveur de l'école moderne. Ainsi, avec le retour au multipartisme dans les années 1990 au Cameroun, il se produit une forte intégration des fils Mbororo dans la plus haute sphère administrative et 
les services de transports interurbains privés avec création des agents de voyage. Ainsi, par endroits, le contrôle de la terre va échapper aux chefs de terre au bénéfice des chefs Mbororo et devenir des sources de litiges entre autochtones et Mbororo.

Progressivement, les fons (« chefs de terre ») ne sont plus tenus informés des transactions foncières pratiquées par des héritiers fonciers surtout chez les familles de notables de Bamenda. Ce détournement des anciennes règles coutumières s'explique par les revendications des grandes familles, descendantes des lignées d'ancêtres locaux et surtout par l'émergence d'une rente foncière. À l'économie foncière, essor démographique, occupation intense des terres et la saturation foncière viennent révéler des conflits fonciers dormants entre les populations.

Les natifs de Mbengwi accusent les Mbororo d'avoir déguerpi et occupé trois de leurs villages (Munam, Mbengeghang, Guneku); les éleveurs Mbororo auraient chassé 300 paysans de leur village qui peut risquer de provoquer une guerre de vengeance entre les ethnies. Un affrontement de cette nature a déjà eu lieu il y a quelques années à Santa, autre localité du NordOuest.

\section{Élevage extensif, saturation foncière dans le Nord-Ouest et départ vers les savanes de Banganté}

Le Nord-Ouest présente des chaines montagneuses (mont Oku, 3000m d'altitude) avec un relief, d'une végétation herbeuse, des cours d'eau et de nombreux lacs cratères (Gwanfogbe et al., 1983). En dehors de la saison pluvieuse (de juillet à octobre), la région offre, tout au long de l'année, un climat propice à l'élevage (Neba, 1999). Dans cette zone, la quantité de fourrage est fonction des pluies. Tout au long de l'année, le bétail se nourrit de graminées (Andropogon gayanus, Setaria pumila, Loudetia togoensis, Sporobolus festivus, Andropogon pseudapricus, Heteropogon contortus) et de ligneux (Anogeissus leiocarpus, Parinari curatellifolia, Monotes kerstingii, Combretum glutinosuum, Cissus cornifolia). Toutefois, le pâturage est menacé de disparition à cause des surcharges en bétail. Les $83 \%$ de cheptel appartiennent au grand Nord Cameroun, les $17 \%$ sont repartis dans les régions du Nord-Ouest, du Sud et de l'Est (Minipa, 2000). La sédentarisation des Mbororo les a amenés à pratiquer aussi l'agriculture. Désormais, les éleveurs sont devenus des agro-éleveurs mais ils doivent se déplacer en période difficile, à la recherche de lieux d'abreuvement et de pâturages.

En effet, l'expansion des surfaces cultivées par les autochtones qui ont remis en cause les accords verbaux d'occupation des années 1960 avec des éleveurs, a réduit les espaces pastoraux occupés par les Mbororo. L'évolution démographique paysanne et la création de plusieurs champs en brousse ont multiplié des conflits entre éleveurs et agriculteurs. Les incidents sont répétés 
pendant les campagnes agricoles entre autochtones-agriculteurs et éleveurs. L'insécurité foncière s'aggrave au Nord-Ouest. Une forte saturation foncière et une grande consommation d'espace par les éleveurs entraînent des conflits fonciers (par exemple aux villages Tourwa, Furu-Wa, Fundong, Kumbo, Mbengwi ...) parfois dramatiques.

\section{L'ampleur des changements fonciers et remise en cause des accords passés dans le Nord-Ouest}

Grâce à l'hospitalité des chefferies (Mankon, Bafut, Bali-Nyongha, Nso, Kom) envers les éleveurs Mbororo dans les années 1920 à 1960, plusieurs d'entre eux ont profité des accords verbaux des chefs de terre pour s'implanter.

Cependant, l'accroissement de la pression démographique actuelle, les investissements agroindustriels (Tea Estate, Magzi, Fainap) par des entrepreneurs dans le Nord-Ouest viennent remettre en cause l'occupation de grands espaces par des Mbororo. La terre, aujourd'hui, n'est en effet plus cédée. Des retraits partiels ou totaux de terres s'opèrent aux dépens de Mbororo de première génération sédentarisés qui avaient cédé une partie des terres à leurs enfants. Les retraits devenant au fil des années de plus en plus fréquents, des tensions foncières prennent plusieurs formes. Ces tensions foncières poussent des familles de la jeune génération Mbororo à quitter le Nord-Ouest en direction de la rive gauche du Noun (années 1980) et de Bangangté (années 1990- jusqu'aujourd'hui).

Cependant, un accès libéral à la terre n'existe pratiquement pas à Bangangté. Dans cet espace arboré et sécurisé, toute occupation volontaire ou libre est exclue, les terres de Bangangté étant immatriculées par les chefs traditionnels. Les terres sont louées et les éleveurs installés sont tous identifiés. Les contrats de location ont une durée le plus souvent de cinq ans renouvelable. Les terres sont matérialisées et immatriculées. La location des terres d'élevage se fait d'abord oralement puis elle est finalisée par écrit entre les Mbororo venus du Nord-Ouest et un notable de la chefferie.

Parfois la location de terre est payée en avance à un prix moyen entre 200000 à $300000 \mathrm{Fcfa} / 400$ ha et accompagné de dons d'animaux à la notabilité. En effet, les éleveurs doivent contribuer en dons d'animaux aux grandes funérailles traditionnelles Bangangté (Medumba). La gestion des terres relève du pouvoir des notables. Les éleveurs sédentaires savent qu'ils occupent le patrimoine foncier des Bangangtés.

\section{Saturation foncière et stratégies endogènes des Mbororo dans le Nord- Ouest vers la région de l'Ouest-Cameroun (Banganté)}

En rappel, les MBororos du Cameroun constituent un groupe ethnique qui parle une même langue et qui fait partie des groupes dits des minorités : 
venus du Nord-Cameroun, sédentarisés, confrontés à une saturation foncière à Bamenda puis disséminés dans toute la région du Nord-Ouest, de l'Ouest et maintenant à Bangangté (département du Ndé).

La nouvelle génération Mbororo est consciente que la terre est finie à Bamenda! Dans une stratégie endogène, elle se lancée à la conquête de nouvelles terres ailleurs. Ainsi, les Mbororo sont maintenant à Bangangté (Ndé) mais le droit foncier traditionnel y est très contraignant. Plus loin, le laisser-faire de l'Etat a facilité le mouvement de colonisation pastorale Mbororo aux abords de la route nationale de Bafia en direction de la région du Centre. Toutefois, les Mbororo évitent d'atteindre la forêt dense, par crainte de la mouche tsé-tsé, dangereuse pour les bovins.

\section{Éveil de l'ethnie Mbororo et difficile revendication d'un droit foncier}

Les travaux de Mouiche (2012) montrent que, sous le parti unique (1966-1990/91), le rôle des Mbororo se limitait aux taxes fiscales. Mais, le retour au multipartisme et leur éducation scolaire vont les pousser à participer aux échéances électorales et dans les prises de decisons. Les éleveurs enquêtés (figure) ont passé un académique et leur éducation a provoqué un éveil chez les jeunes éleveurs.

Aujourd'hui, les Mbororo sont définitivement intégrés (Mimche et al., 2006). Ainsi, les Mbororo se sont appuyés sur la législation foncière de 1974 pour se faire aider à travers l'association MBOSCUDA par le Ministère de l'administration territoriale et de la décentralisation en vue d'immatriculer plus de 300 ha de terres à leurs noms dans le «pays » Bamoun (villages Didango, Koutaba, Macha) et ils ont désormais un chef (lamido) traditionnel de 3é degré reconnu par l'Etat dans ces villages d'éleveurs.

Dès les années 1990, le chef traditionnel de Bangangté a accepté l'installation de Mbororo, en leur accordant une hospitalité contrôlée et surveillée. Les Mbororo sont installés en des villages (Tchoudim-matap, Banoudim, Tchounoun, Sarki, Projet, Bangang-Fokam ...), dans un espace immatriculé avec des bornes. Le bornage a été effectué par le chef de terre sous le contrôle des notables, pour éviter que les Mbororo deviennent autochtones. Pourtant, les zones délimitées pour l'élevage par le chef de terre n'empêchent pas les animaux de provoquer des dégâts aux cultures. Dès lors, les conflits fonciers entre agriculteurs et Mbororo sont souvent récurrents. 


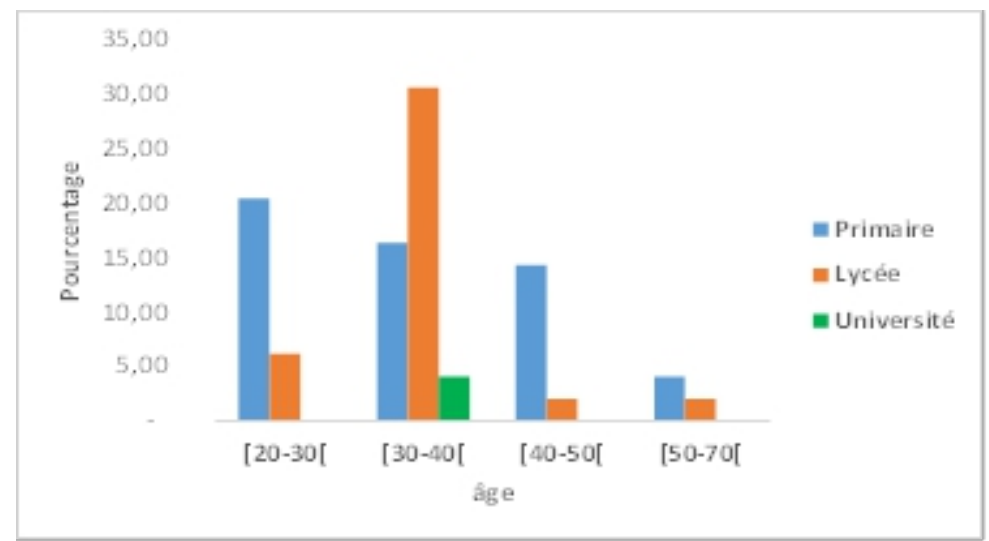

Figure 1. Niveau académique des éleveurs enquêtés

Il ressort de cette figure que l'ensemble des éleveurs ont fait l'école primaire avant d'abandonner, on enregistre un faible pourcentage $(04,08 \%) \mathrm{du}$ niveau académique supérieur. Néamoins, cette éducation scolaire facilite la bonne compression de la législation foncière nationale chez les éleveurs.

\section{Savane arborée de Bangangté : difficile terre d'accueil des éleveurs Mbororo}

La commune de Bangangté avec une population d'environ 300000 habitants s'étend sur une superficie de $800 \mathrm{~km}^{2}$ et comporte sept villages : Bangangté, Bangoulap, Bangoua, Bamena, Batchingou, Bangang-Fokam et Bahouoc. Chacun de ces villages a à sa tête un chef supérieur qui est soit du 1er, 2ème ou 3ème degré (Lebel \& Pontié., 2011). La maîtrise des terres est détenue par les anciens et les notables qui sont les gardiens et héritiers fonciers. À côté de proches de forêt (primaire et secondaire) et des forêts galeries en bordure des cours d'eau, la savane est la formation végétale dominante dans cet espace. Cette savane remplace la forêt primaire par suite de défrichage et de feux de brousse chaque saison. Deux types de forêts existent dans cette zone : les forêts naturelles (forêts sacrées) et les forêts reboisées (eucalyptus, pin...).

Cet espace est très convoité par la nouvelle génération d'éleveurs Mbororo en quête de pâturages. Dans la racine de mobilité et sédentarisation, leur installation va commencer dans les années 1990, suivie de la difficile négociation avec la chefferie. Ici, le mode d'installation de Mbororo est diffèrent de celui du Nord-Ouest, l'éleveur Mbororo ne disposant que d'un droit d'usage foncier. Les chefs de terre de Bangangté ont très vite compris l'économie foncière et limité l'arrivée des étrangers. Pour assurer une sécurité foncière aux nouveaux venus, la plupart des chefs traditionnels disent qu'ils sont juste gardiens du patrimoine de la population autochtone. Il faut noter qu'au début des années 1990 et conformément à la coutume des Bangangté, 
c'est le chef de terre (souvent le notable, le plus proche du village) qui décide de l'octroi des parcelles foncières aux autochtones comme aux Mbororo (allochtones) et ces notables en informent rapidement le chef supérieur.

Les paysans autochtones ayant sollicité, directement ou par un intermédiaire, le notable donateur de terres, ce dernier juge la capacité et l'intérêt agricole de la parcelle que le paysan compte mettre en valeur. Celuici ne tarde pas à obtenir l'exploitation d'une portion de terroir d'étendue délimitée. En échange, le paysan s'engage à verser une partie de sa récolte lors des grandes cérémonies traditionnelles de fin d'année à la chefferie.

Les espaces réservés à l'élevage sont des sommets de reliefs et l'espace impropre à l'agriculture. Ils sont octroyés aux Mbororo par accord verbal et la superficie est délimitée. Il leur est toutefois demandé de ne pas conduire les animaux dans les bas-fonds très proches du fleuve Noun, où la culture maraichère est très développée, afin d'éviter des conflits entre éleveurs et agriculteurs.

Alors que l'hospitalité des chefs de terre était très grande dans la région du Nord-Ouest, mais, qu'à Bangangté, tout est contrôlé et l'espace étant immatriculé comme patrimoine des ancêtres réservé aux autochtones, les terres sont sécurisées avec les bornes. Le contrôle de la terre n'échappe pas aux chefs de terre. Le terroir est anciennement partagé de façon approximative entre les petites chefferies. Les chefs de terre sont toujours tenus informés des transactions foncières avec un nouveau venu. Les règles coutumières sont très respectées par tous.

A Bangangté, la terre n'est pas cédée mais louée aux éleveurs. Les contrats de location, pour une durée de cinq ans renouvelable, sont conclus oralement mais parfois par écrit entre le notable et le Mbororo. Les éleveurs savent qu'ils sont sur la terre du chef de Banganté. Des retraits poussent des familles entières, installées depuis les années 1990, à quitter la zone pour rechercher des terres libres vers la region du Centre (Bafia). Les persécutions des Mbororo sont souvent récurrentes. Le nouveau Mbororo à de la peine à s'installer.

Bien que le nombre de Mbororo soient estimé à une centaine de familles, la cohabitation avec les autochtones s'est détériorée ces dernières années par suite de xénophobie (Tableau 1). Les autochtones remettent en cause les accords verbaux d'installation des éleveurs, ils n'acceptent plus leur présence dans cette localité. Des plantations agricoles viennent d'être créées dans les pâturages d'éleveurs par des paysans autochtones et certains bestiaux sont abattus de façon massive provocante des conflits entre acteurs. 
Tableau 1. Typologie de conflits fonciers enregistrés

\begin{tabular}{|c|c|c|c|c|}
\hline âge & $\begin{array}{c}\text { contre notabilité } \\
\%\end{array}$ & $\begin{array}{c}\text { contre paysans } \\
\%\end{array}$ & $\begin{array}{c}\text { entre éleveurs } \\
\%\end{array}$ & Total \\
\hline [20-30[ & 22,45 & 2,04 & 10,20 & 34,69 \\
\hline [30-40[ & 18,37 & 2,04 & 4,08 & 24,49 \\
\hline$[40-50[$ & 20,41 & 6,12 & 2,04 & 28,57 \\
\hline [50-70[ & 10,20 & 2,04 & - & 12,24 \\
\hline Total & 71,43 & 12,24 & 16,33 & 100 \\
\hline
\end{tabular}

Il ressort de ce tableau que le conflit foncier est très développé entre éleveurs et la notabilité foncière $(71 \%)$ et faiblement enregistré entre paysan-éleveurs $(12 \%)$.

\section{Mbororo, chefferie, Etat, ONG et la question foncière à Bangangté}

Installés dans un espace immatriculé (borné) et aménagé, les Mbororo sont controlés. Dans un contexte de conflits fonciers récurrents, d'empiètements sur les terres imparties aux Mbororo par les paysans et d'accusations contre ces éleveurs considérés comme des étrangers, les autochtones revendiquent leur terre : "Comment le chef Banganté peut-il se donner abusivement le pouvoir d'installer les Mbororo à celui qu'il appelle chef de quartier (ardo-Mbororo) ? or, le territoire qui n'est pas le sien? Il n'a qu'à rester là où le pouvoir de l'Etat lui confère certaines prérogatives. Il ne doit en aucun cas aller au-delà des limites imposées. Cette terre appartenait à nos ancêtres locaux dont nous sommes des cohéritiers et le chef doit rester seulement le gardien du patrimoine ».

Les plaintes des Mbororo se multiplient et sont déposées auprès des autorités, en signe de leurs inquiétudes, aux chefs traditionnels et autorités administratives et, au-delà, auprès de la société civile : Fronline Fighters for Citizen's Interest (FFCI) dont la vocation est de défendre les droits des citoyens et enfin au Centre International Pour la prévention des Génocides et des Catastrophe Environnementales (CIPGECE).

Du côté de l'administration locale, trois types de conflits ont été identifiés. Le rejet des éleveurs Mbororo, les conflits entre agriculteurs et éleveurs qui sont provoqués par des dégâts aux cultures et par l'obstruction des voies d'accès aux couloirs de pâturages et de l'exploitation de aux cours d'eau comme source d'alimentation. Il faut aussi noter que la remise en cause des accords antérieurs entre autochtones et Mbororo (accord verbal) est une autre cause de conflits.

\section{Conflit foncière et médiation administrative}

Dans ce contexte d'instabilité croissante, le préfet du departement du Ndé, en compagnie du maire, ainsi que du chef supérieur Bangangté, a organisé une rencontre pour dénoncer publiquement l'attitude xénophobe de quelques citoyens «tapis dans l'ombre». Ils ont tenu à rassurer la 
communauté Mbororo qui s'est sentie inquiétée par des tracts anonymes en circulation contre eux. "Les MBororos sont ici chez eux et doivent se sentir libres d'aller et venir » a tenu à préciser le préfet. Une position appuyée par les autorités municipales et traditionnelles (schéma). Cependant, l'incident de Bangangté est simplement la partie émergée d'un iceberg.

Le mal vivre entre les MBororos et les autochtones est de l'avis de plusieurs observateurs, plus profond. La première offensive contre eux est venue, il y a quelques années, un membre appartenant à la couche aisée qui est un riche local. Ce dernier s'est en effet installé dans la zone agro-pastorale de Tchada, à Baleng region de 1'Ouest. " Cette zone est interdite aux cultures pérennes. Quand ce membre s'y est installé, c'était pour cultiver du maïs et d'autres cultures saisonnières. Mais son projet a très tôt été dépassé, à cause de violences et du rejet local».

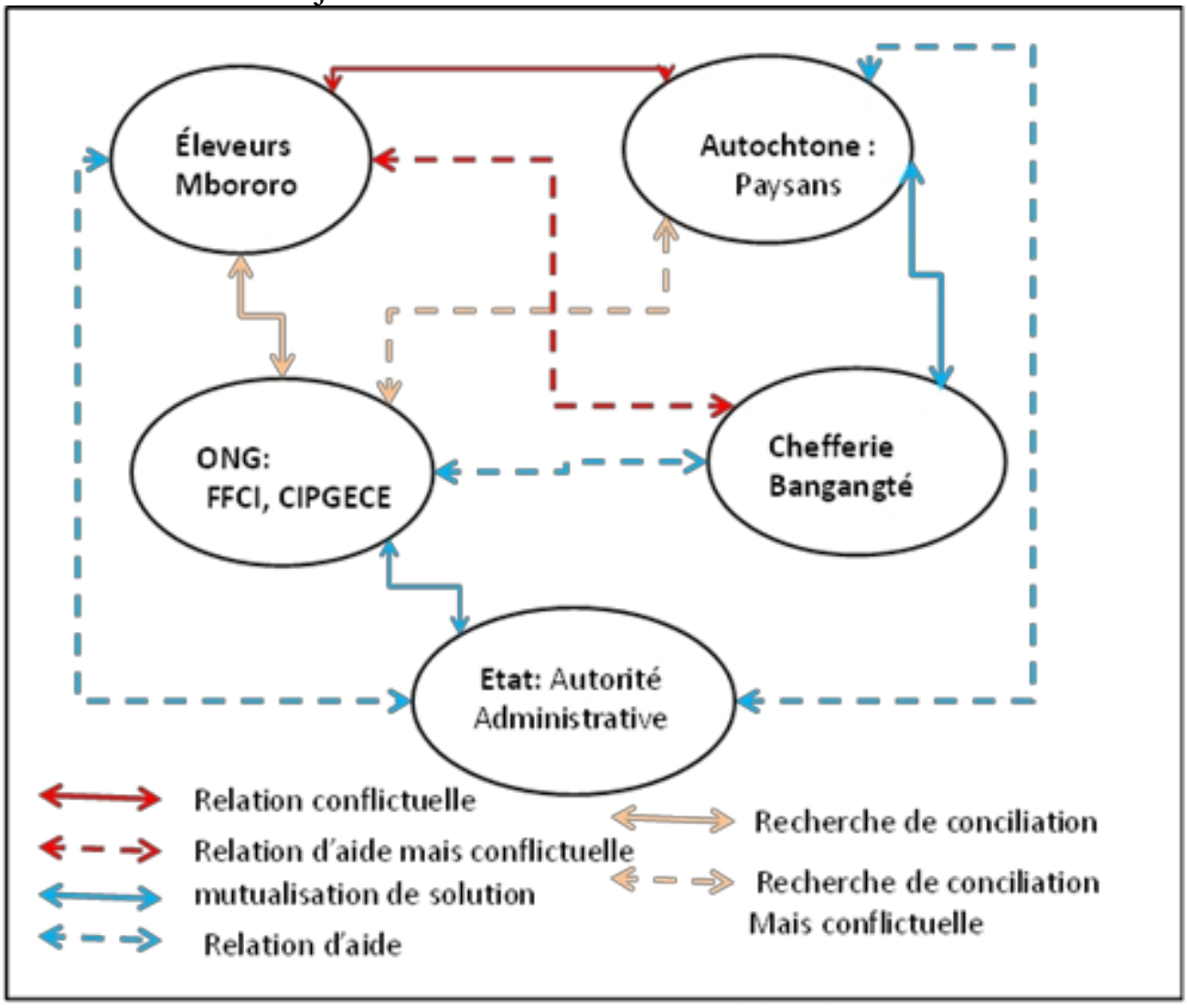

Source : Mfewou A, $(2018$

Figure 1. Analyse de conflit foncier et difficile conciliation à Bangangté

Il ressort de l'analyse qu'à Bangangté, l'accueil des Mbororo se fait de façon différente des autres zones du Cameroun. L'occupation de terres, librement et sans limite n'y existe pratiquement pas. Le domaine national 
occupé par les éleveurs est immatriculé par la chefferie avec des bornes. L'ampleur des remises en cause qui passent par des formes diverses de renégociation avec les autorités locales et les ONG montrent que les Mbororo n'ont qu'un droit d'usage. Le schéma témoigne des difficultés des actions de conciliation et de la complexité foncière du jeu d'acteurs. Plusieurs d'entre eux sont impliqués dans les opérations de contrôle des terres face à la nouvelle génération de Mbororo «éveillés » qui, de leur côté, brandissent à chaque fois la législation foncière camerounaise de 1974. Le politique semble être absent dans l'accompagnement d'acteurs.

\section{Conclusion}

A l'Ouest-Cameroun, les savanes de Bangangté ne sont vides d'occupation qu'apparent. Ce capital foncier, bien que rélevant du domaine national, reste entre les mains de la chefferie traditionnelle économiquement. Il est considéré comme le patrimoine de la population dont le chef reste le gardien.

$\mathrm{Au}$ Nord-Ouest, les éleveurs Mbororo avaient acquis des grandes superficies de terre grâce à une hospitalité et de l'accord verbal des chefs coutumiers. Mais, la difficile sédentarisation se présente différemment à Bangangté (Ouest-Cameroun). Or, la logique migratoire des éleveurs Mbororo avec leur cheptel, la dynamique des agriculteurs et la croissance démographique de la population paysanne sont ici indissociables car le développement local tient une place importante dans l'accroissement de la population et de vivre ensemble.

Cet élevage représente une part essentielle de la stratégie de commercialisation bovine locale. Les boucheries (bovines) sont déjà très fréquentes à Bangangté et appartiennent à la couche moyenne locale. Ces bouchers s'approvisionnent en animaux chez les éleveurs Mbororo local.

Les notables fonciers qui contrôlent l'espace ne souhaitent qu'accorder qu'une hospitalité surveillée et limitée aux Mbororo. Ceux-ci ne sont que des usufruitiers et doivent garder en mémoire qu'ils sont sur le patrimoine des Bangangtés. Ici, le contrôle foncier est bien décentralisé entre les mains de notables.

Au contraire, pour les éleveurs Mbororo, la terre étant un attribut divin dont le chef de terre n'est que le dépositaire, elle ne se refuse pas. À travers l'éducation et leur association (MBOSCUDA), les Mbororo «éveillés » estiment qu'ils sont installés sur le domaine de l'Etat régi par la législation foncière de 1974. Si la perméabilité du système foncier de Bangangté a permis d'accueillir partiellement ce flux migratoire par une installation contrôlée, il faut signaler la puissance et l'action des notabilités foncières qui sont très informées de la présence des étrangers sur leur sol. Par contre, l'Etat est absent de l'encadrement et la délimitation d'espaces réservés à l'élevage, de couloirs 
de circulation des animaux et d'autres espaces attribués à l'agriculture. L'Etat lui-même y est pour beaucoup. Il manque jusqu'ici une véritable politique d'aménagement de l'espace et de prévision pour se doter d'une politique d'accompagnement de mobilités des éleveurs. Or, il est necessaire que le politique assure les éléveurs par une législation adequate à un accés dans $1^{\prime}$ espace, à une sédentarisation parfaite et à une sécurisation fonciere. Cette sécurisation foncière va aussi assurer un meilleur élevage et 1 'optimisation de la productivité du troupeau.

\section{References:}

1. Boutrais, J. (1984). Entre nomadisme et sédentarité: Les Mbororo à l'ouest du Cameroun. 1984. ISO. 690.

2. Boutrais, J. (1992). Zébus, mouches tsé-tsé et techniciens en Adamaoua camerounais. Robineau C.(ed. SC.): Les terrains du développement; approches pluri-disciplinaires des économies du Sud, Paris, Orstom, Didactiques, 235-252.

3. Courade, G. (2007). Géographe ORSTOM-IRD dans une afrique en mouvement. Revue Tiers Monde, (3), 553-572.

4. Dongmo, A.L., Djamen, P., Vall, E., Koussou, M.O., \& Doubangolo, $\mathrm{C}$ et al. (2012). «Du nomadisme à la sédentarisation », Revue d'ethnoécologie [En ligne], 1 | 2012.

5. Exchange, F. (2010). West Africa in general. Journal of African economies (Ox ford), 19, 5.

6. Gallais, J. (1982). Pôles d'États et frontières en Afrique contemporaine. Cahiers d'outre-mer, 35(138), 103-122.

7. Gwanfogbe, M., Meligui, A., Moukam, J., \& Nguoghia, J. (1983). Geography of Cameroon. Hong Kong: Macmillan Education. ISBN $\underline{0-}$ 333-36690-5.

8. Lebel, A. \& Pontié, E. (2011). «Bangangté », in Le Cameroun aujourd'hui, Éditions du Jaguar, Paris, 2011, p. 114 (ISBN 978-286950-464-6).

9. Mfewou, A. \& Nchoutnji, I. (2014). Migration maraîchère et enjeux fonciers à la frontière de la ville de Yaoundé (Cameroun). ISO.690.

10. Mimche, H. \& Michaela, P. (2006). Quand les immigrés se font autochtones Immigration et dynamique d'insertion des Mbororo et insécurité foncière à l'Ouest-Cameroun. Communication, Burkina Faso, l'Asile du Sud : Afrique, Méditerranée. 5-7 juin 2006.

11. Mouiche, I. (2012). Démocratisation et intégration sociopolitique des minorités ethniques au Cameroun: entre dogmatisme du principe majoritaire et centralité des partis politiques. CODESRIA, Conseil pour le développement de la recherche en sciences sociales en Afrique. ISO. 690. 
12. Neba, A. (1999). Modern Geography of the Republic of Cameroon. Bamenda: Neba Publishers.

13. Ngengong, T. E. (2007). From Friends to Enemies: Inter-Ethnic conflict amongst the Tikars of the Bamenda Grassfields (North West Province of Cameroon) C. 1950-1998.

14. Pelican, M. (2011). Mbororo on the move: from pastoral mobility to international travel. Journal of Contemporary African Studies, 29(4), 427-440.

15. Pemunta, V. N. \& Brice, A. A. (2013). Socio-spatial occupation, conflict and humanitarian assistance for Bororo cross-border migrants in east Cameroon. International Journal of Development Issues, 12(3), 271-288.

16. Sado, A. T. (2010). Application de la méthode d'évaluation contingente à la mesure de la demande et à la planification des investissements scolaires dans les zones sous-scolarisées. Cas des populations Mbororo du Nord-Ouest. ISO. 690.

17. Tallet, B. (2003). Comment gérer un «espace fini »? Nouveaux enjeux fonciers en zone de colonisation agricole dans l'Ouest du Burkina Faso. Savanes africaines : Actes du colloque, mai 2002, Garoua, Cameroun. Prasac, N'Djamena, Tchad - Cirad, Montpellier, France.

18. Vallois, H. V. \& Marquer, P. (1980). Notes ethno-démographiques sur les Pygmées de la République Populaire du Congo et de ses zonesfrontières avec les Républiques Centrafricaine et Gabonaise. Bulletins et Mémoires de la Société d'Anthropologie de Paris, 7(2), 109-123. 